\title{
Indice di massa corporea e rischio di 22 tipi di cancro: uno studio di coorte basato su una popolazione di 5 milioni e 240 mila adulti del Regno Unito
}

\author{
Michele Marinò
}

Pubblicato online: 4 dicembre 2014

C Springer International Publishing AG 2014

\section{Commento a:}

Body-mass index and risk of 22 specific cancers: a population-based cohort study of 5.24 million UK adults.

K. Bhaskaran, I. Douglas, H. Forbes, I. dos-Santos-Silva, D.A. Leon, L. Smeeth. Lancet (2014) 384:755-765

La relazione tra sovrappeso/obesità e cancro è da anni oggetto di controversie. Benché vi sia un generale accordo sul fatto che un indice di massa corporea (BMI) elevato predisponga allo sviluppo di alcuni tumori, fino a questo studio non erano disponibili studi su casistiche sufficientemente ampie da poter trarre conclusioni certe sull' argomento.

In questo brillante studio condotto nel Regno Unito, veniva valutata la frequenza di cancro in base al BMI su un'enorme casistica derivata dal Clinical Practice Research Datalink. Venivano analizzati i dati di ben 5,24 milioni di persone, di cui 166.955 avevano almeno un cancro. Elevati valori di BMI risultavano essere associati significativamente con 17 dei 22 tipi di cancri osservati, con livelli di significatività dipendenti dal tipo di cancro. In particolare, ogni in- cremento di BMI di $5 \mathrm{~kg} / \mathrm{m}^{2}$ si associava in maniera lineare con il cancro del corpo dell'utero (hazard ratio [HR] 1,62, 99\% CI 1,56-1,69; $p<0,0001$ ), della colecisti (HR 1,31, CI $1,12-1,52 ; p<0,0001)$, del rene (HR 1,25, CI 1,171,$33 ; p<0,0001$ ), della cervice uterina (HR 1,10, CI 1,031,$17 ; p=0,00035$ ), della tiroide (HR 1,09, CI 1,00-1,19; $p=0,0088$ ), e con la leucemia (HR 1,09, CI 1,05-1,13; $p \leq 0,0001)$. Il BMI era inoltre positivamente associato con il cancro del fegato (HR 1,19, CI 1,12-1,27), del colon (HR 1,10 , CI 1,07-1,13), delle ovaie $(1,09$, CI 1,04-1,14) e con il cancro della mammella post-menopausale (HR 1,05, CI 1,03-1,07) (tutti con una significatività con $p<0,0001$ ). Complessivamente, veniva stimato che un incremento del BMI di $1 \mathrm{~kg} / \mathrm{m}^{2}$ nella popolazione generale del Regno Unito determinerebbe un incremento dei casi di cancro di 3790 unità.

Questo importantissimo studio dimostra inequivocabilmente che l'obesità o comunque l'incremento del BMI si associa a un aumentato rischio di cancro e conferma la necessità di una stretta sorveglianza socio-sanitaria sullo stile di vita della popolazione, volta a prevenire e curare i disturbi del peso corporeo.
M. Marinò $(\varangle)$

U.O. Endocrinologia 1, Università di Pisa, Pisa, Italia

e-mail: michele.marino@med.unipi.it 